\title{
Piezoelectric Response Evaluation of ZnO Thin Film Prepared by RF Magnetron Sputtering
}

\author{
Da-Long Cheng ${ }^{1}$, Kuo-Sheng Kao ${ }^{1}$, Chia-Hua Liang ${ }^{2}$, Yu-Che Wang ${ }^{1}$, Yu-Chieh Chen ${ }^{1}$, Wei-Che Shih ${ }^{3}$ \\ and Leong-Perng Chan ${ }^{4,5, a}$ \\ ${ }^{1}$ Department of Computer and Communication, Shu-Te University, Taiwan \\ ${ }^{2}$ Department of Cosmetic Science, Chia Nan University of Pharmacy and Science, Taiwan \\ ${ }^{3}$ Department of Electrical Engineering, National Sun Yat-Sen University, Kaohsiung, Taiwan \\ ${ }^{4}$ Institute of Clinical Medicine, Kaohsiung Medical University, Kaohsiung, Taiwan \\ ${ }^{5}$ Department of Otolaryngology-Head and Neck Surgery, Kaohsiung Medical University Hospital, Kaohsiung Medical \\ University, Kaohsiung, Taiwan
}

\begin{abstract}
The most important parameter of piezoelectric materials is piezoelectric coefficient (d33). In this study, the piezoelectric $\mathrm{ZnO}$ thin films were deposited on the $\mathrm{SiNx} / \mathrm{Si}$ substrate. The 4 inches substrate is diced into $8 \mathrm{~cm} \times 8 \mathrm{~cm}$ piece. During the deposition process, a zinc target (99.999 wt\%) of 2 inches diameter was used. The vertical distance between the target and the substrate holder was fixed at $5 \mathrm{~cm}$. The piezoelectric response of zinc oxide $(\mathrm{ZnO})$ thin films were obtained by using a direct measurement system. The system adopts a mini impact tip to generate an impulsive force and read out the piezoelectric signals immediately. Experimentally, a servo motor is used to produce a fixed quantity of force, for giving an impact against to the piezoelectric film. The $\mathrm{ZnO}$ thin films were deposited using the reactive radio frequency (RF) magnetron sputtering method. The electric charges should be generated because of the material's extrusion. This phenomenon was investigated through the oscilloscope by one shot trigger. It was apparent that all $\mathrm{ZnO}$ films exhibit piezoelectric responses evaluated by our measurement system, however, its exhibit a significant discrepancy. The piezoelectric responses of $\mathrm{ZnO}$ thin film at various deposition positions were measured and the crystal structures of the sputtering pressure were also discussed. The crystalline characteristics of $\mathrm{ZnO}$ thin films are investigated through the XRD and SEM. The results show the $\mathrm{ZnO}$ thin film exhibits good crystalline pattern and surface morphology with controlled sputtering condition. The $\mathrm{ZnO}$ thin films sputtered using 2 inches target present various piezoelectric responses. With the exactly related position, a best piezoelectric response of $\mathrm{ZnO}$ thin film can be achieved.
\end{abstract}

\section{Introduction}

Piezoelectric materials have been attracting considerable attention because of their potential applications for acoustic devices [1], micro motors[2], AFM cantilevers [3], UV detectors [4-5] or chemical sensors [6]. The most important parameter of piezoelectric materials is piezoelectric coefficient (d33). Conventionally, the techniques for piezoelectric coefficient measurement can be

\footnotetext{
${ }^{\mathrm{a}}$ Corresponding author: oleon24@yahoo.com.tw
} 
divided into two categories: direct measurement and indirect measurement.In this study, we takes focus on the direct measurement method of piezoelectric coefficient by using a homemade stable force generator. Firstly, a servo motor is used to produce a fixed quantity of force, for giving animpact against to the piezoelectric film. The electric charges should be generated because of the material's extrusion. This phenomenon will be investigated through the oscilloscope by one shottrigger. The influences caused from various film thickness have been studied. The responses of the piezoelectric film are expected to be monitored under the condition with quantitative force. Finally, a reliable platform of piezoelectric coefficient measurement will be established by suitable mechanical modificationdesign.

\section{Experimental}

\subsection{Thin Film process}

In this study, the piezoelectric $\mathrm{ZnO}$ thin films were deposited on the $\mathrm{SiNx} / \mathrm{Si}$ substrate. The 4 inches substrate is diced into $8 \mathrm{~cm} \times 8 \mathrm{~cm}$ piece. During the deposition process, a zinc target $(99.999 \mathrm{wt} \%)$ of 2 inches diameter was used. The vertical distance between the target and the substrate holder was fixed at $5 \mathrm{~cm}$. In order to illustrate the sample position, we defined the value of $\mathrm{R} / \mathrm{r}$ in the sputtering chamber, where $r$ is the radius of zinc target and $R$ is the distance between the sample and the substrate holder centre, as shown in Fig. 1. By varying the value of $\mathrm{R} / \mathrm{r}$, the influence of the geometry inside the chamber on the thin film properties can be realized.

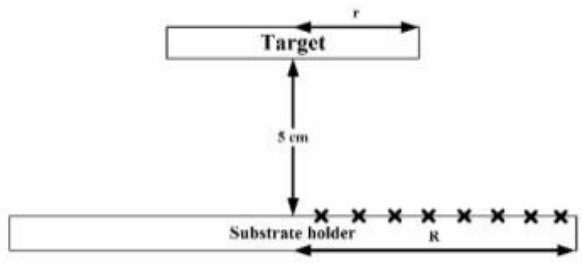

Figure 1. Schematic illustration of the sample position in the sputtering chamber.

Deposition parameters of various conditions were show in Table 1 . $\mathrm{ZnO}$ thin films were deposited using the reactive radio frequency $(\mathrm{RF})$ magnetron sputtering method. In order to obtain the high $\mathrm{c}$ axis orientation $\mathrm{ZnO}$ thin films characteristics, two-step $\mathrm{ZnO}$ thin films deposition method was adopted to improve $\mathrm{ZnO}$ thin films characteristics. The $\mathrm{ZnO}$ thin films characteristics were determined by X-ray diffraction (XRD) using a Bruker D8 Advance with $\mathrm{CuK} \alpha$ radiation. Cross-sectional morphologies of the $\mathrm{ZnO}$ thin films were observed by a scanning electron microscopy (JEOL6700 FESEM).

Table 1. Deposition parameters

\begin{tabular}{|c|c|}
\hline Targets & Zinc (99.999 wt. \%) 2 inch \\
\hline Substrate & $\mathrm{SiNx} / \mathrm{Si}$ \\
\hline Base pressure (Torr) & $<1 \times 10^{-6}$ \\
\hline $\begin{array}{c}\text { Working pressure } \\
\text { (mTor) }\end{array}$ & $10,15,25$ \\
\hline Sputtering power (W) & 85 \\
\hline Deposition time (min) & 60 \\
\hline $\begin{array}{c}\text { Substrate temperature } \\
\left({ }^{\circ} \mathrm{C}\right)\end{array}$ & R. T. \\
\hline $\mathrm{O}_{2} /\left(\mathrm{O}_{2}+\mathrm{Ar}\right)(\%)$ & 75 \\
\hline
\end{tabular}




\subsection{Measurement Instrument}

Figure 2 shows the experimental setup to fabricate the direct measurement system of piezoelectric response. The setup consists of a mini impact probe, a cantilever, a servo motor, an oscilloscope, a translation stage, and specimens to be tested. An impact probe driving by a servo motor connected with a stainless steel rod is submitted close to the sample surface and in contact with substrate. The probe with a metal tip acts perpendicularly to the thin film's surface to generate electrical pulse. In turn, due to the piezoelectric effect, the mechanical displacement of the film produces output electrical signal at the tip. In the meantime, the direct piezoelectric voltage can be measured by the impact probe.
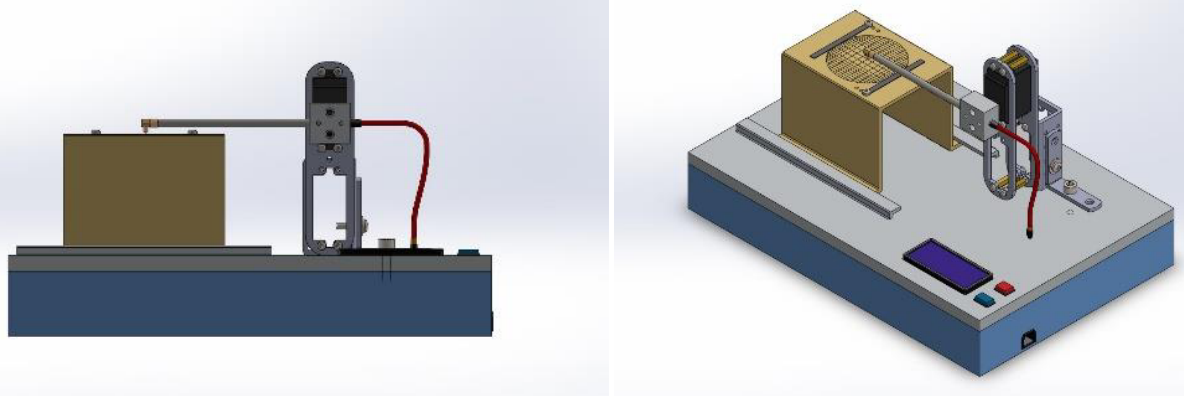

Figure 2. Experimental setup.

\section{Results}

\subsection{Influence of the sputtering positions}

As is shown in Fig. 3, a concentric ring pattern of rainbow colours forms because the different wavelengths of light interference at different film thicknesses. An obviously Newton's rings presented that a clear and definite discrepancy of thin film thickness is without a doubt. It can be concluded that the thickness of the $\mathrm{ZnO}$ film is decreased as the sample is placed far away from the target. In other words, the insufficient supplement of the ions results in the lack of atoms for the $\mathrm{ZnO}$ growth, leading to a thinner film thickness.

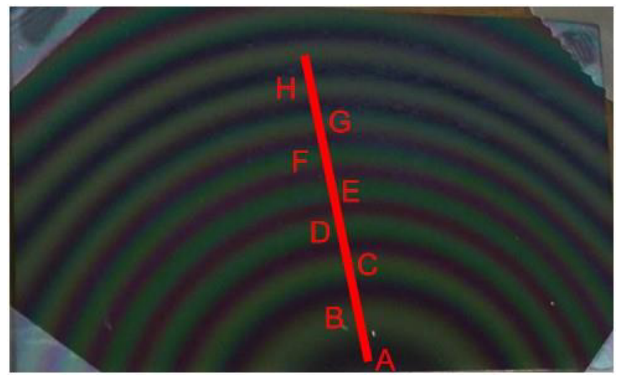

Figure 3. The top-view of a half substrate

\subsection{Influence crystal structure of the sputtering pressure}

Firstly, the sputtering pressure was varied to determine the optimal growth parameters of the $\mathrm{ZnO}$ thin films. The sputtering parameters were shown in table 1, the substrate temperature was set room temperature and sputtering pressure was set to $10 \mathrm{mTorr}, 15 \mathrm{mTorr}$ and $20 \mathrm{mTorr}$ to investigate $\mathrm{ZnO}$ thin films of crystal structure. Fig. 4 shows XRD pattern of $\mathrm{ZnO}$ thin films deposition on the $\mathrm{SiNx} / \mathrm{Si}$ substrate, revealing the excellent $\mathrm{ZnO}(002)$ orientated crystals around $34.5^{\circ}$. Even though XRD of the 
sputtering pressure at $15 \mathrm{mTorr}$ was presented the highly c-axis oriented $\mathrm{ZnO}(002)$, the SEM of Fig. 5(b) shows good surface structure. Figure 5 exhibited $\mathrm{ZnO}$ thin films surface morphologies with various sputtering pressure, the Fig. 5(a) and (c) show disorder crystal structure. Besides, the Fig. 5(b) presented the uniform cobblestone crystal and identical grain. It exhibited the thin film has higher the $\mathrm{ZnO}(002)$ diffraction peak, the strongly c-axis (002) orientation of a piezoelectric thin films that has the higher electromechanical coupling coefficient [7].

As a result, the optimal sputtering pressure of $15 \mathrm{mT}$ orr, RF power of $85 \mathrm{~W}$ and $\mathrm{O}_{2} / \mathrm{O}_{2}+\mathrm{Ar}$ ratio of $75 \%$ were used to deposit $\mathrm{ZnO}$ thin films. The study result shows a strong c-axis-orientated crystallization, uniform grain size, well-textured columnar structures of the $\mathrm{ZnO}$ thin film.

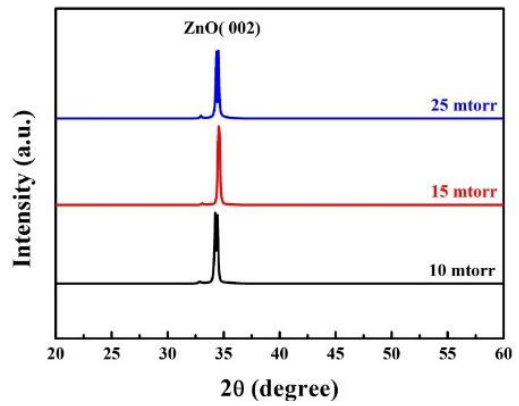

Figure 4. The XRD pattern with various sputtering pressure of (a)10 mTorr (b)15 mTorr (c)20 mTorr

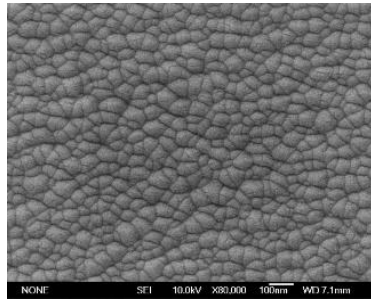

(a)

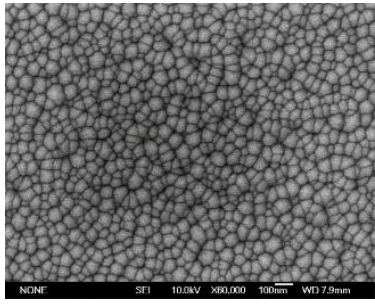

(b)

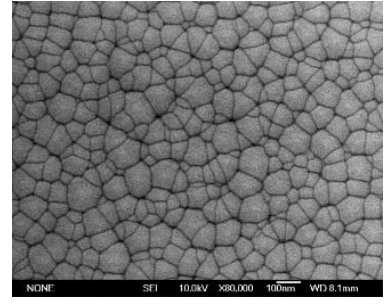

(c)

Figure 5. The surface morphologies with various sputtering pressure of (a) 10 mTorr (b) 15 mTorr (c) 20 mTorr

\subsection{Piezoelectric response with related position}

Figure 6 shows the piezoelectric responses of these $\mathrm{ZnO}$ films at various positions. The experimental data are directly captured from the direct measurement system. It is apparent that all $\mathrm{ZnO}$ films exhibit piezoelectric responses evaluated by our measurement system, however, its exhibit a significant discrepancy. Figure 7 shows the variations in the peak voltages of the responses as a function of sample position. From our observation, the increase in the peak voltage of $\mathrm{ZnO}$ films can be mainly attributed to the increment in the film's quality.

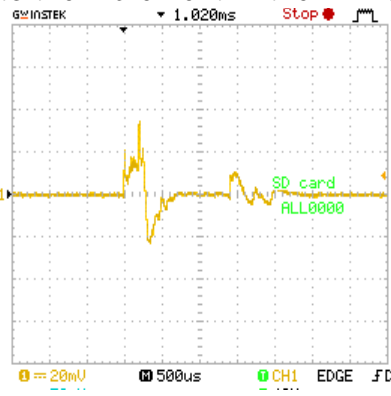

(A)

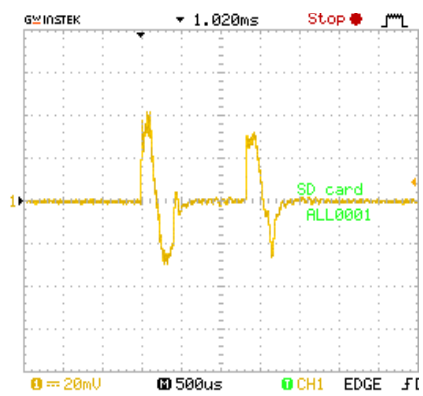

(B) 


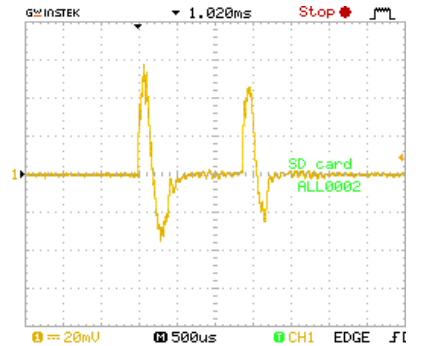

(C)

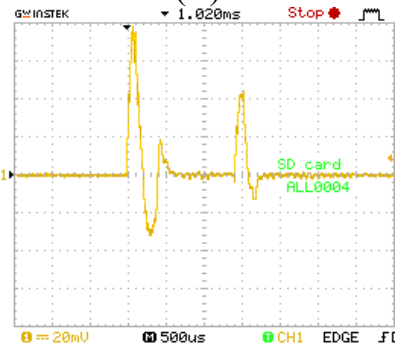

(E)

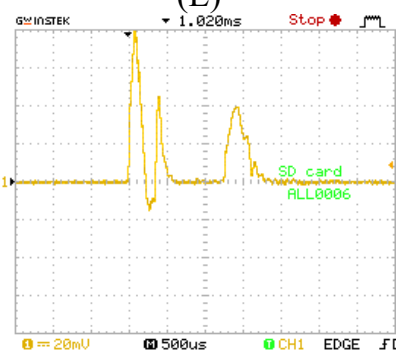

(G)

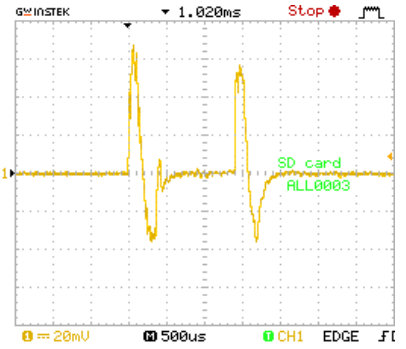

(D)

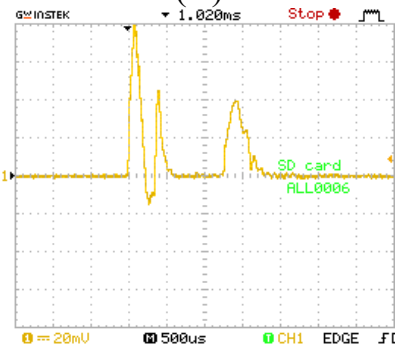

(F)

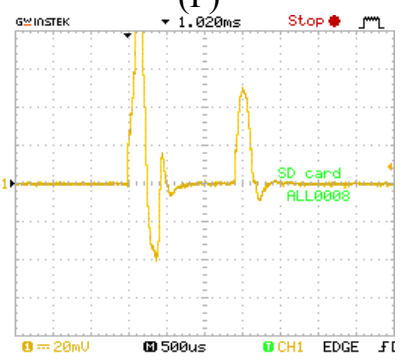

(H)

Figure 6. Piezoelectric response at various positions

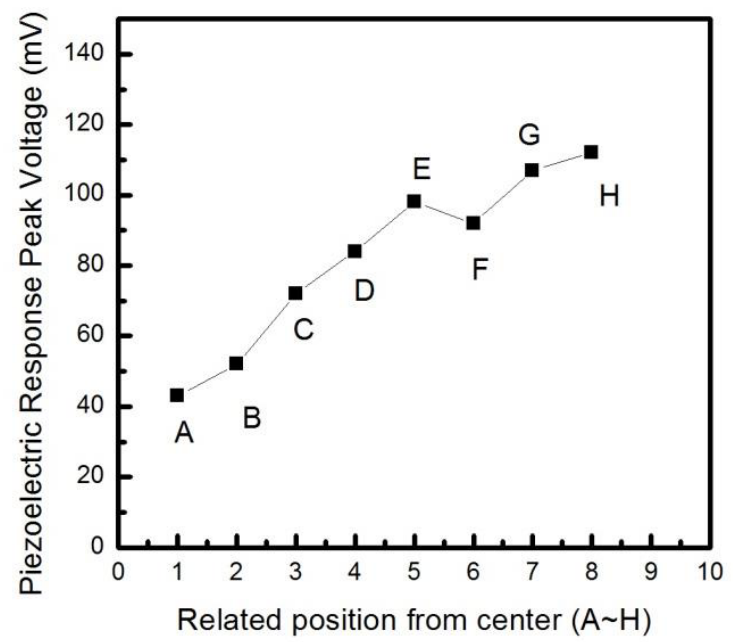

Figure 7. Peak voltage of piezoelectric response with related position 


\section{Conclusions}

In this study, the $\mathrm{ZnO}$ thin films are deposited on $\mathrm{SiNx} / \mathrm{Si}$ substrate. The crystalline characteristics of $\mathrm{ZnO}$ thin films are investigated through the XRD and SEM. The results show the $\mathrm{ZnO}$ thin film exhibits good crystalline pattern and surface morphology with controlled sputtering condition. The $\mathrm{ZnO}$ thin films sputtered using 2 inches target present various piezoelectric responses. With the exactly related position, a best piezoelectric response of $\mathrm{ZnO}$ thin film can be achieved.

\section{Acknowledgment}

The authors thank the Ministry of Science and Technology of the Republic of China, Taiwan, for financially supporting this research under contract numbers MOST 105-2632-E-366 -001.

\section{References}

1. R.C. Lin, Y.C. Chen, K.S. Kao, Appl. Phys. A Mater. Sci. Process. 89,475 (2007)

2. M.-A. Dubois, P. Muralt, IEEE Trans. Ultrason., Ferroelect., Freq. Contr. 45, 1169(1998)

3. T. Itoh, T. Suga, Appl. Phys. Lett. 64, 37 (1994)

4. C. L. Wei, Y. C. Chen, K. S. Kao, K. T. Wu, D. L. Cheng, P. T. Hsieh, J. Micro/Nanolith. MEMS MOEMS, 9, 031009(2010)

5. K. S. Kao, W. C. Shih, W. T. Ye, D. L. Cheng, Thin Solid Films, 605, 77(2016)

6. J. Xia, S. Burns, M. Porter, T. Xue, G. Liu, R. Wyse, C. Thielen, IEEE Inter-national Frequency Symposium, 879 (1995)

7. W. Water, S.Y. Chu, Materials Letters, 55, 67 (2002) 